\title{
Commensal symbiosis between a Lactococcus lactis strain and an Enterococcus mundtii strain increases cell yield in constituted broth
}

\author{
H. Kimoto-Nira, ${ }^{1}$ H. Ohmori, and C. Suzuki \\ NARO Institute of Livestock and Grassland Science, Ikenodai 2, Tsukuba, Ibaraki 305-0901, Japan
}

\begin{abstract}
To exert their beneficial effects, probiotics need to survive in the stringent conditions of the gastrointestinal tract. Symbiosis between different bacteria is a potential way of enhancing this survival. In developing new probiotic cultures, we investigated the synergic effect between Enterococcus mundtii IFO 13712 and 7 strains of Lactococcus lactis, many of which are widely used as starter bacteria for making dairy products and have probiotic properties. The growth yield of a mixed culture of L. lactis strain Y and IFO 13712 in de Man, Rogosa, and Sharpe broth was greater than that of a single culture. Supernatant from culture of strain IFO 13712 enhanced the growth of strain Y, but that of strain Y did not enhance the growth of strain IFO 13712. This commensalism phenomenon was confirmed by using a simpler tryptone-yeast extract-glucose (TYG) broth. Increased cell yield in mixed culture of the 2 strains compared with single cultures was observed in TYG broth in the presence of both Tween 80 and citrate but not in TYG broth alone or TYG broth containing either Tween 80 or citrate. Thus, the Tween 80 and citrate in the broth contributed to the commensalism. Metabolite analysis revealed that ethanol production in the co-metabolism of glucose and citrate by strain $\mathrm{Y}$ was suppressed by mixed culture in TYG broth containing Tween 80 and citrate, compared with that in TYG broth containing citrate alone. The mechanism supporting the observed commensal symbiosis between strains Y and IFO 13712 was the increase in availability of glucose for lactate production by strain Y because, in glycolysis, the pathway from glucose to lactate is energic, whereas the pathway from glucose to ethanol is not. Whether growth stimulation of strain Y by mixing it with IFO 13712 in milk products will enhance the survival of strain $\mathrm{Y}$ in the intestine remains to be elucidated.
\end{abstract}

Received May 24, 2012.

Accepted July 8, 2012.

${ }^{1}$ Corresponding author: anne@affrc.go.jp
Key words: enterococci, lactococci, starter culture, symbiosis

\section{INTRODUCTION}

Probiotics are defined by the Food and Agriculture Organization and World Health Organization as "live microorganisms which, when administered in adequate amounts, confer a health benefit on the host." It has been suggested that bacteria ingested as probiotics cannot affect the intestinal environment unless their populations reach a minimal threshold of $10^{6}$ to $10^{8}$ $\mathrm{cfu} / \mathrm{g}$ in intestinal content (Marteau and Rambaud, 1993). For the bacteria to accumulate in the intestinal tract, bacterial growth needs to be enhanced.

Prebiotics have been defined as "nondigestible food ingredients that beneficially affect host health by selectively stimulating the growth and/or activity of one or a limited number of bacteria in the colon" (Gibson and Roberfroid, 1995). Lactobacilli and bifidobacteria are among the intestinal bacteria stimulated by prebiotics (Haarman and Knol, 2006; Iwata et al., 2009). Prebiotics combined with probiotic bacteria in the form of synbiotics are capable of improving the gastrointestinal health of humans.

Enterococci are bacteria that occur naturally in the intestinal tract, and most strains therefore tolerate the conditions found the intestinal tract. Some strains of enterococci have been isolated from fermented milk products (Ao et al., 2012; Faye et al., 2012) and have probiotic activities, such as the ability to reduce numbers of Salmonella enteritidis and coliforms (Bhardwaj et al., 2010) and to exert a bactericidal effect on diarrheagenic Escherichia coli (Miyazaki et al., 2010). In contrast, lactococci are not intestinal bacteria but can tolerate the conditions found in the intestinal tract (Klijn et al., 1995). Lactococci are widely used as starter bacteria in the manufacture of dairy products such as fermented milk and cheese. Furthermore, they have probiotic activities such as the improvement of skin condition in mice (Kimoto-Nira et al., 2007) and improvement of lipid metabolism in rats (Lee et al., 2005). Despite the ability of prebiotics to enhance the 
Table 1. Enterococcus and Lactococcus strains examined

\begin{tabular}{llll}
\hline Species & Strain & Origin & Source $^{1}$ \\
\hline Enterococcus mundtii & 13712 & Silkworm & IFO \\
Lactococcus lactis ssp. lactis & 19435 & Unknown & ATCC \\
L. lactis ssp. lactis & 527 & Raw milk & Laboratory collection \\
L. lactis ssp. lactis & G50 & Raw grass & Laboratory collection \\
L. lactis ssp. lactis & $\mathrm{H} 46$ & Kimchi & Laboratory collection \\
L. lactis ssp. lactis bv. diacetylactis & $\mathrm{N} 7$ & Commercial cheese starter & Laboratory collection \\
L. lactis ssp. lactis bv. diacetylactis & $\mathrm{H} 59$ & Kimchi & Laboratory collection \\
L. lactis ssp. lactis bv. diacetylactis & $\mathrm{Y}$ & Commercial cheese starter & Laboratory collection \\
\hline
\end{tabular}

${ }^{1} \mathrm{IFO}=$ Institute for Fermentation Osaka (Osaka, Japan); ATCC = American Type Culture Collection (Manassas, VA).

value of enterococci or lactococci as probiotic bacteria, few reports on this topic have been published. In the present study, we attempted to increase growth of enterococci or lactococci by mixed cultivation of these species.

In the dairy industry, mixed cultures, rather than pure cultures, of lactic acid bacteria are typically used for making fermented milk products because pure strains often grow slowly in milk and mixed cultures can improve the flavor (Kothari et al., 1971; Frengova et al., 2000). Mixed starter cultures of lactococci with other lactic acid bacteria such as Leuconostoc spp. and lactobacilli are used, rather than single cultures. In addition, Picon and Nunez (2007) reported growth stimulation of a proteinase-positive Lactococcus lactis strain by a proteinase-negative L lactis strain. If certain substances, such as prebiotics, released from enterococci or lactococci can be used to promote the growth of these probiotic species, then mixed fermentation of these 2 genera (Lactococcus and Enterococcus) in milk products might enhance their cell counts and thus their intestinal survival. Here, we investigated the growth stimulation of lactococci by enterococci as a model of this interaction.

\section{MATERIALS AND METHODS}

\section{Strains and Culture Conditions}

The 8 strains of lactic acid bacteria used included Enterococcus mundtii IFO 13712 (Institute for Fermentation Osaka, Osaka, Japan), L. lactis ssp. lactis ATCC 19435 (American Type Culture Collection, Manassas, VA), and 6 strains from our laboratory collection (Table 1). With the exception of strain ATCC 19435, the strains of lactococci were potential candidate probiotic bacteria based on previous reports from our laboratory and others. Lactococcus strain G50 induces the production of cytokines from a macrophage-like cell line, and oral administration of strain G50 to mice suppresses the production of serum immunoglobulin E
(Kimoto et al., 2004a). Oral administration of L. lactis ssp. lactis 527 and L. lactis ssp. lactis bv. diacetylactis N7 improves lipid metabolism in rats (Lee et al., 2005). Lactococcus lactis ssp. lactis bv. diacetylactis $\mathrm{Y}$ induces cytokine production by a macrophage-like cell line (Kimoto et al., 2004a). Lactococcus lactis ssp. lactis H46 can remove cholesterol from broth (Kimoto et al., 2004b), whereas L. lactis ssp. lactis bv. diacetylactis H59 produces bacteriocin (T. Okamoto, M. Nomura, H. Kimoto, M. Kobayashi, K. Mitani, and T. Narita, NARO Institute of Livestock and Grassland Science, Ibaraki, Japan, unpublished data). The strains were maintained by subculture of $1 \%$ inocula in deMan, Rogosa, and Sharpe (MRS) broth (Becton Dickinson, Sparks, MD). Cultures were incubated for $18 \mathrm{~h}$ at $30^{\circ} \mathrm{C}$ (for lactococci) or $37^{\circ} \mathrm{C}$ (for E. mundtii IFO 13712). The cultures were stored at $4^{\circ} \mathrm{C}$ between transfers and subcultured once before use.

\section{Growth in Single and Mixed Cultures}

For each experiment, a tube containing $20 \mathrm{~mL}$ of MRS or tryptone-yeast extract-glucose (TYG) broth was inoculated with E. mundtii IFO 13712 at $10^{7} \mathrm{cfu} /$ $\mathrm{mL}$ or the desired strain of L. lactis at $10^{7} \mathrm{cfu} / \mathrm{mL}$, or both. The TYG broth consisted of $0.5 \%$ tryptone (Becton Dickinson), 0.5\% yeast extract (Wako Chemical, Osaka, Japan), and $1.0 \%$ glucose supplemented with $1 \%$ sodium succinate and $1 \%$ sodium chloride. The $\mathrm{pH}$ of the TYG broth was adjusted to $\mathrm{pH} 6.86$. Single and mixed cultures were incubated for $24 \mathrm{~h}$ at $37^{\circ} \mathrm{C}$. To simulate the conditions in the intestine, these experiments were performed at $37^{\circ} \mathrm{C}$, not at the optimal growth temperature $\left(30^{\circ} \mathrm{C}\right)$ for lactococci. At the end of incubation, bacterial growth was determined by measuring the optical density of the cultures at a wavelength of $620 \mathrm{~nm}\left(\mathbf{O D}_{620}\right)$ with a Spectronic 20 spectrophotometer (Bausch \& Lomb, Rochester, NY) against an uninoculated broth blank. Bacterial growth was also determined from the dry weight of the cells. For measurement of dry weight, cells in the cultures 
were collected by centrifugation $(2,800 \times g, 20 \mathrm{~min})$ at $4^{\circ} \mathrm{C}$, washed twice with distilled water, and dried at $105^{\circ} \mathrm{C}$ for $4 \mathrm{~h}$.

\section{Contact-Free Coculture on Bacterial Growth}

For this assay, a cell-culture insert $(0.4-\mu \mathrm{m}$ pore size, Falcon type 3095, Becton Dickinson Labware, Oxnard, CA) was placed in 24-well tissue culture plates (Falcon type 2046). Growth medium was added to the culture well and the culture insert to a volume of $0.5 \mathrm{~mL}$. Bacterial cells were seeded at a concentration of $10^{7} \mathrm{cfu} /$ $\mathrm{mL}$ to the culture well and the insert. The plate was incubated at $37^{\circ} \mathrm{C}$. After $24 \mathrm{~h}$ of incubation, bacterial growth in the culture plate and culture insert was determined with a spectrophotometer (DU-640, Beckman Coulter Inc., Brea, CA), as described above. In this system, the 2 strains of bacteria do not contact each other, but bacterial metabolites from one strain are able to reach the cells of the other strain.

In an another experiment using culture plates, Lactococcus strain $\mathrm{Y}$ was cultured with the supernatant from E. mundtii IFO 13712-inoculated MRS broth or with the supernatant from strain-Y-inoculated MRS broth for $24 \mathrm{~h}$ at $37^{\circ} \mathrm{C}$. These supernatants were adjusted to $\mathrm{pH} 6.5$ with $1 \mathrm{~N} \mathrm{NaOH}$ and sterilized by filtration $(0.2 \mu \mathrm{m})$. Bacterial growth was quantified as described above.

\section{Substrate and Metabolite Analysis}

Culture supernatants were stored at $-20^{\circ} \mathrm{C}$ until the time of analysis for substrate and product. Residual glucose in the broth was measured enzymatically with a Glucose CII test kit (Wako). Lactate, citrate, acetate, and formate in the broth after culture were quantified by using a postcolumn HPLC system (LC-UV, Shimadzu, Tokyo, Japan) equipped with a Shim-pack SCR-102H column $(8 \mathrm{~mm} \times 300 \mathrm{~mm}$; Shimadzu $)$ fitted with a SCR-102H guard column and a CDD-10Avp conductivity detector (Shimadzu). The mobile phase contained $5 \mathrm{~m} M$ p-toluenesulfonic acid. The postcolumn buffer solution was composed of $5 \mathrm{~m} M p$-toluenesulfonic acid, $100 \mu M$ EDTA, and $20 \mathrm{~m} M$ Bis-Tris. Ethanol was quantified with an F-kit (Boehringer, Mannheim, Germany). Acetoin was measured by the method of Westerfeld (1945), and $\mathrm{CO}_{2}$ was not quantified.

Cellular sugar content was determined by the phenolsulfate method (Dubois et al., 1956). Cells were harvested and washed 3 times with distilled water and then resuspended in water. The cell suspensions were used to analyze the sugar content and measure the dry weight. Milligrams of glucose per milligram of dry cell weight were calculated, and the results were expressed as percentage glucose.

\section{Statistical Analysis}

Data are expressed as means or means \pm standard deviation from 3 samples and a representative from 2 independent experiments. Statistical analysis was performed on the differences between single and mixed cultures by using the $t$-test, and $P<0.05$ was considered statistically significant.

\section{RESULTS AND DISCUSSION}

\section{Interaction of E. mundtii IFO 13712 with Lactococci to Increase Cell Yield}

Seven strains of lactococci were cocultured with $E$. mundtii IFO 13712 in MRS broth. The $\mathrm{OD}_{620}$ of a mixed culture of Lactococcus strain Y or N7 with strain IFO 13712 in MRS broth was markedly greater than that for each single Lactococcus and Enterococcus culture (Table 2). Because the increase in growth of strain Y in the presence of strain IFO 13712 was higher than that of strain N7 with strain IFO 13712, we selected strain Y for subsequent experiments. The dry weight of strains IFO 13712 and $\mathrm{Y}$ in mixed culture $(900.0 \pm 1.50 \mathrm{mg} / \mathrm{L})$ was significantly higher than that of strain IFO 13712 $(780.0 \pm 17.6 \mathrm{mg} / \mathrm{L} ; P=0.0012)$ or strain $\mathrm{Y}(795.6 \pm$ $20.4 \mathrm{mg} / \mathrm{L} ; P=0.0037)$ individually.

We also investigated the interaction between strain $\mathrm{Y}$ and Enterococcus faecalis ATCC strains 27274 (obtained from ATCC) and IFO 12964 and IFO 12968 (ob-

Table 2. Bacterial growth in single and mixed cultures (in de Man, Rogosa, and Sharpe broth) of Enterococcus mundtii IFO 13712 and Lactococcus strains

\begin{tabular}{lcc}
\hline & \multicolumn{2}{c}{ OD $_{620}$} \\
\cline { 2 - 3 } Bacterial strain & Single & Mixed \\
\hline IFO 13712 & 1.54 & $\mathrm{ND}^{2}$ \\
ATCC 19435 & 1.01 & 1.35 \\
527 & 1.40 & 1.40 \\
G50 & 1.60 & 1.56 \\
H46 & 1.74 & 1.74 \\
N7 & 1.56 & 1.72 \\
H59 & 1.83 & 1.87 \\
Y & 1.49 & 1.73 \\
\hline
\end{tabular}

${ }^{1}$ Enterococcus mundtii IFO 13712 and Lactococcus strains (see Table 1 for species designations) were cultured in single and mixed fermentations (mixed cultures included the listed Lactococcus strain plus IFO 13712). Values were determined by measuring the optical density (OD) at a wavelength of $620 \mathrm{~nm}$ after a $24-\mathrm{h}$ incubation at $37^{\circ} \mathrm{C}$. Each value is the mean of 3 samples.

${ }^{2}$ Not done. 
tained from IFO). Mixed cultures of each of these Enterococcus strains and strain $\mathrm{Y}$ did not increase growth compared with that of each single culture (data not shown), suggesting that the symbiosis is not specific to Enterococcus species.

The sugar contents of strain IFO 13712 or strain Y cells grown in MRS broth were compared with that of mixed-culture cells. The sugar content of strain IFO 13712 cells (dry weight) was $21.2 \pm 2.40 \%$, that of strain Y cells was $22.6 \pm 0.80 \%$, and that of the mixedculture cells was $20.8 \pm 1.55 \%$; these values did not differ significantly. This result suggests that polysaccharides, such as those in the capsules of bacterial cells, were not responsible for the increase in dry weight after mixed culture of these strains.

\section{Contact-Free Coculture of Strain IF013712 or Strain $Y$}

Table 3 shows the growth of strain IFO 13712 or strain Y mixed with either strain in a cell-free culture system. Growth of strain $\mathrm{Y}$ in the culture insert and culture plate was enhanced by coculture with a cellfree filtrate of strain IFO 13712, whereas the growth of strain IFO 13712 in the culture insert and culture plate was not enhanced by coculture with a cell-free filtrate of strain Y.

Culture filtrate from MRS culture of strain IFO 13712 or $\mathrm{Y}$ was prepared, the $\mathrm{pH}$ was adjusted, and the filtrate was added to MRS broth inoculated with strain Y. Growth was determined by time interval, and a growth curve was obtained. The maximal growth rate of strain Y mixed with cell-free filtrate of strain IFO $13712(0.173 \pm 0.010 / \mathrm{h})$ was significantly $(P<0.01)$ higher than that of strain $\mathrm{Y}$ mixed with cell-free filtrate

Table 3. Bacterial growth in single and mixed cultures (in de Man, Rogosa, and Sharpe broth) of Enterococcus mundtii IFO 13712 and Lactococcus lactis ssp. lactis bv. diacetylactis strain $\mathrm{Y}$ in culture inserts (upper side) and culture plates (lower side) ${ }^{1}$

\begin{tabular}{llc}
\hline Culture method & Strain & OD $_{620}$ \\
\hline Culture insert & IFO 13712 & 1.53 \\
Culture plate & IFO 13712 & 1.38 \\
Culture insert & Y & 1.98 \\
Culture plate & IFO 13712 & 1.31 \\
Culture insert & IFO 13712 & 1.39 \\
Culture plate & Y & 1.59 \\
Culture insert & Y & 1.78 \\
Culture plate & Y & 1.47
\end{tabular}

${ }^{1}$ Strains IFO 13712 and Y were cultured in single and mixed fermentation. Values were determined by measuring the optical density (OD) at a wavelength of $620 \mathrm{~nm}$ after a $24-\mathrm{h}$ incubation at $37^{\circ} \mathrm{C}$. Each value is the mean of 3 samples. of strain Y $(0.112 \pm 0.004 / \mathrm{h})$. This interaction between strains IFO 13712 and Y was indicative of commensalism - one strain producing or releasing compounds that promote the growth of the other (Meers 1973).

de Man, Rogosa, and Sharpe broth has many components. We therefore used TYG broth (a simpler medium) in our subsequent experiments to attempt to reveal the mechanism by which coculture of strains IFO 13712 and Y showed enhanced growth yield compared with each single culture. In TYG broth, a mixed culture of strains Y and IFO 13712 had a greater growth yield compared with a single culture of strain Y but not IFO 13712 (Table 4) - an additive, but not synergistic, effect. The fact that the MRS broth contained $0.1 \%$ Tween 80 , and TYG broth did not, led to us hypothesize that Tween 80 was involved in the commensal symbiosis. To test the hypothesis, we prepared TYG broth with $0.1 \%$ Tween 80. A mixed culture of strains Y and IFO 13712 in TYG broth containing $0.1 \%$ Tween 80 did show an increase in growth yield over a single culture of strain Y but not strain IFO 13712 (Table 4); again, an additive, but not synergistic, effect. Therefore, we needed to study other components of the medium.

de Man, Rogosa, and Sharpe broth contains $0.2 \%$ ammonium citrate, whereas TYG broth does not. To clarify the effect of citrate on commensal symbiosis, we first added 0.2 or $0.4 \%$ ammonium citrate to TYG broth and adjusted it to $\mathrm{pH} 6.86$ with $1 \mathrm{~N} \mathrm{HCl}$. At $0.2 \%$ ammonium citrate, mixed culture of strains $\mathrm{Y}$ and IFO

Table 4. Bacterial growth in single and mixed cultures of Enterococcus mundtii IFO 13712 and Lactococcus lactis ssp. lactis bv. diacetylactis strain $\mathrm{Y}$ in different media ${ }^{1}$

\begin{tabular}{lcc}
\hline & $\begin{array}{c}\text { Dry weight } \\
(\mathrm{mg} / \mathrm{L})\end{array}$ & $P$-value \\
\hline Medium and culture & & \\
TYG broth & $691.1 \pm 3.85$ & $<0.01$ \\
IFO 13712 & $591.1 \pm 7.70$ & $<0.01$ \\
Y & $644.4 \pm 10.2$ & \\
Mixed & & \\
TYG plus 0.1\% Tween 80 & $575.6 \pm 10.2$ & 0.19 \\
IFO 13712 & $355.6 \pm 21.4$ & $<0.05$ \\
Y & $517.8 \pm 51.7$ & \\
Mixed & & \\
TYG plus 0.4\% ammonium citrate & $720.0 \pm 3.67$ & $<0.01$ \\
IFO 13712 & $755.6 \pm 3.85$ & 0.13 \\
Y & $746.7 \pm 6.67$ & \\
Mixed & & \\
TYG plus 0.1\% Tween 80 and $0.4 \%$ & & $<0.01$ \\
ammonium citrate & & \\
IFO 13712 & $693.3 \pm 13.3$ & \\
Y & $642.2 \pm 13.9$ & $<0.01$ \\
Mixed & $851.1 \pm 10.2$ & \\
\hline
\end{tabular}

$\overline{{ }^{1} \text { Strains IFO } 13712 \text { and } \mathrm{Y} \text { were cultured in single and mixed fermenta- }}$ tions in different broths (tryptone-yeast extract-glucose, TYG, supplemented with Tween 80, ammonium citrate, or both) for $24 \mathrm{~h}$ at $37^{\circ} \mathrm{C}$. Values were expressed as dry weight, as described in the Materials and Methods. Each value is the mean \pm SD from 3 samples. A $t$-test was applied to single versus mixed fermentation in each broth. 
13712 did not show an increase in growth yield over either single culture (data not shown). Similarly, at $0.4 \%$, mixed culture of strains Y and IFO 13712 did not show an increase in growth yield over either single culture (Table 4). Finally, TYG broth with both $0.1 \%$ Tween 80 and 0.2 or $0.4 \%$ ammonium citrate was prepared. With $0.1 \%$ Tween 80 and $0.4 \%$ ammonium citrate, mixed culture of strains Y and IFO 13712 showed a significant increase in growth yield over each single culture (Table 4); this increase was not seen with the broth containing $0.1 \%$ Tween 80 and $0.2 \%$ ammonium citrate (data not shown). These results suggest that both $0.1 \%$ Tween 80 and $0.4 \%$ ammonium citrate had a positive influence on growth of these strains in mixed culture.

\section{Analysis of Metabolites from Pure and Mixed Fermentation of Strains IFO 13712 and $Y$}

Lactococcus and Enterococcus are homofermentative lactic acid bacteria. Metabolism in homolactic acid bacteria leads to $>90 \%$ conversion of sugars to lactate. Under certain conditions, such as galactose utilization (Garrigues et al., 1997) and aerobic conditions (Smart and Thomas, 1987), other metabolites besides lactate, such as acetate, formate, ethanol, and $\mathrm{CO}_{2}$, are produced; this process is generally called mixedacid fermentation. Lactococcus lactis ssp. lactis bv. diacetylactis, a citrate-utilizing lactic acid bacterium, is a component of mixed starter cultures used in the dairy industry to manufacture fermented products. The breakdown of citrate results in the production of $\mathrm{CO}_{2}$, acetate, and acetoin. Flavor compounds such as acetoin have been reported in dairy products (Callon et al., 2005; Chammas et al., 2006). For example, diacetyl and its reduced form, acetoin, are of interest for their organoleptic properties and are responsible for notes of a fermented buttery flavor.

We analyzed the metabolites from single and mixed cultures of the test strains in each broth (Table 5). In E. mundtii IFO 13712, lactate was the main end product from glucose in the presence or absence of citrate; the citrate was not consumed. In Lactococcus strain Y cultured in TYG broth or TYG plus Tween 80, lactate, acetate, formate, and ethanol were produced from glucose; acetoin was detected in the TYG broth but not in the TYG plus Tween 80 broth. Notably, although Lactococcus strain $\mathrm{Y}$ showed mixed-acid fermentation of glucose, it is unlikely that the type of fermentation in glycolysis was responsible for the symbiosis between strains IFO 13712 and Y. We ruled out this possibility because we also observed an increase in the OD value when strain IFO 13712 was cultured with N7 (Table 2), but strain N7 is a glucose homofermentative bacterium (Kimoto et al., 1999).

In co-metabolizing glucose and citrate, strain Y produced lactate, acetate, formate, ethanol, and acetoin (Table 5). In mixed culture, the main metabolic organic acids were also lactate, acetate, formate, and ethanol. We observed differences in lactate, formate, and ethanol production between mixed culture in TYG containing citrate and mixed culture in TYG containing both Tween 80 and citrate. In TYG containing

Table 5. Fermentation products from single and mixed cultures of Enterococcus mundtii IFO 13712 and Lactococcus lactis ssp. lactis bv. diacetylactis strain $\mathrm{Y}$ in different media $^{1}$

\begin{tabular}{|c|c|c|c|c|c|c|c|}
\hline Medium and culture & \multicolumn{2}{|c|}{ Consumed $(\mathrm{m} M)$} & \multicolumn{5}{|c|}{ Metabolite $(\mathrm{m} M)$} \\
\hline \multicolumn{8}{|l|}{ TYG broth } \\
\hline $\mathrm{Y}$ & 14.4 & 0.00 & 21.4 & 7.85 & 13.0 & 33.7 & 0.34 \\
\hline Mixed & 21.8 & 0.00 & 45.6 & 3.66 & 4.78 & 19.7 & 0.40 \\
\hline \multicolumn{8}{|c|}{ TYG plus $0.1 \%$ Tween 80} \\
\hline Mixed & 22.3 & 0.00 & 45.3 & 1.23 & 1.44 & 10.8 & 0.26 \\
\hline \multicolumn{8}{|c|}{ TYG plus $0.4 \%$ ammonium citrate } \\
\hline IFO 13712 & 29.8 & 0.00 & 60.1 & 0.86 & 1.76 & 6.50 & 0.75 \\
\hline Y & 18.1 & 18.6 & 26.2 & 36.8 & 23.5 & 41.4 & 0.50 \\
\hline Mixed & 24.6 & 18.6 & 38.4 & 35.0 & 20.6 & 40.4 & 0.64 \\
\hline \multicolumn{8}{|c|}{$\begin{array}{l}\text { TYG plus } 0.1 \% \text { Tween } 80 \text { and } 0.4 \% \\
\text { ammonium citrate }\end{array}$} \\
\hline
\end{tabular}

${ }^{1}$ Strains IFO 13712 and Y were cultured in single and mixed fermentations in different broths (tryptone-yeast extract-glucose, TYG, supplemented with Tween 80 , ammonium citrate, or both) for $24 \mathrm{~h}$ at $37^{\circ} \mathrm{C}$. Each value is the mean of 3 samples.

${ }^{2}$ Not detected. 
citrate, lactate was produced partly from glucose, and the ratio of lactate produced to glucose consumed was 1.56; acetate, formate, and ethanol were produced from citrate and glucose. In TYG containing Tween 80 and citrate, the ratio of lactate to glucose was 1.77 ; the amounts of acetate, formate, and ethanol produced from citrate and glucose were less than that produced in mixed culture in TYG containing citrate. Considering that an increase in cell yield in mixed culture was observed only in the TYG containing both Tween 80 and citrate, increased availability of glucose for lactate production by strain Y must have been the mechanism supporting the observed commensal symbiosis between strains Y and IFO 13712. We arrived at this conclusion because in glycolysis, the pathway from glucose to lactate is energic, whereas the pathway from glucose to ethanol is not. The mechanism by which ethanol production was suppressed by coculture of strains IFO 13712 and $\mathrm{Y}$ in TYG broth containing Tween 80 and citrate is not clear.

An increase in cell yield in mixed cultures of the 2 strains was observed only in TYG containing both Tween 80 and citrate. This finding brings up 2 questions. First, what is the role of Tween 80 in the broth in this symbiosis? Tween 80 is a nonionic detergent. We found that the cell yield of strain IFO 13712 or Y was lower in TYG broth containing Tween 80 in plain TYG broth. It is likely that symbiosis occurred in the presence of some growth suppression. Second, what is the role of the citrate in the broth in this symbiosis? Growth stimulation by citrate has been observed in $L$. lactis ssp. lactis bv. diacetylactis (Cogan, 1981; Starrenburg and Hugenholtz, 1991; Kimoto et al., 1999). In consideration of the molecular energetics of biochemical pathways, the concept developed by Bauchop and Elsden (1960) on yield of cells per mole of energy source or substrate has been widely used (Loubiere et al., 1992; Russell and Cook, 1995). We calculated the molar growth yield from glucose $\left(\mathbf{Y}_{\mathbf{G}}\right)$ for each strain and for mixed culture in each broth (Table 6). In TYG broth containing both Tween 80 and citrate, the $\mathrm{Y}_{\mathrm{G}}$ of the mixed culture did not differ from that of strain IFO 13712 , but it was significantly $(P<0.05)$ lower than that of strain $Y$. The growth activation observed in Leuconostoc spp. by citrate is related to activation of the energetic acetate kinase pathway under sugar and citrate co-metabolism (Schmitt et al., 1990; Schmitt and Divies, 1991). In our study, the $Y_{G}$ values from strain Y grown on TYG broth and on TYG with $0.4 \%$ citrate did not differ (Table 6). This result indicates that strain $Y$ was not able to use citrate as an energy source in the presence of glucose: the energetic pathway in mixed culture was glycolysis alone, under glucose and citrate co-metabolism.
Table 6. Molar growth yields from glucose $\left(\mathrm{Y}_{\mathrm{G}}\right)$ in single and mixed cultures of Enterococcus mundtii IFO 13712 and Lactococcus lactis ssp. lactis bv. diacetylactis strain $\mathrm{Y}$ in different media

\begin{tabular}{lcc}
\hline Medium and culture $^{1}$ & $\mathrm{Y}_{\mathrm{G}}{ }^{2}$ & $P$-value \\
\hline TYG broth & & \\
IFO 13712 & $27.6 \pm 1.38$ & 0.22 \\
Y & $41.5 \pm 6.22$ & 0.07 \\
Mixed & $29.7 \pm 1.99$ & \\
TYG plus 0.1\% Tween 80 & $24.1 \pm 0.52$ & 0.65 \\
IFO 13712 & $33.4 \pm 5.57$ & 0.07 \\
Y & $23.3 \pm 2.67$ & \\
Mixed & $24.2 \pm 0.77$ & $<0.05$ \\
TYG plus 0.4\% ammonium citrate & $42.0 \pm 4.31$ & $<0.05$ \\
IFO 13712 & $30.4 \pm 2.21$ & \\
Y & & \\
Mixed & & \\
TYG plus 0.1\% Tween 80 and 0.4\% & $22.6 \pm 1.21$ & 0.14 \\
ammonium citrate & $40.5 \pm 4.16$ & $<0.05$ \\
IFO 13712 & $24.3 \pm 0.99$ & \\
M Mixed &
\end{tabular}

${ }^{1}$ Strains IFO 13712 and Y were cultured in single and mixed fermentations in different broths (tryptone-yeast extract-glucose, TYG, supplemented with Tween 80, ammonium citrate, or both) for $24 \mathrm{~h}$ at $37^{\circ} \mathrm{C}$. Each value is the mean $\pm \mathrm{SD}$ from 3 samples. A $t$-test was applied to single versus mixed fermentation in each broth.

${ }^{2}$ The $\mathrm{Y}_{\mathrm{G}}$ values were calculated by dividing dry weight by glucose consumed.

\section{CONCLUSIONS}

Lactococci are frequently used in the manufacture of dairy products such as cheese and fermented milk. A trial was conducted on a certain strain of E. mundtii to evaluate it as a probiotic and its growth in milk (Botes et al., 2008). We propose the development of new, mixed starter cultures for dairy products by using lactococci and enterococci. Here, we observed enhancement of cell yield by coculture of $L$. lactis strain $\mathrm{Y}$ and E. mundtii IFO 13712. This is the first report on symbiosis between the 2 species. We need to determine whether the survival of strain $\mathrm{Y}$ in vivo is enhanced by mixing this strain with E. mundtii IFO 13712 in milk culture, for use as a probiotic.

\section{ACKNOWLEDGMENTS}

The authors thank C. Koitabashi, X. Y. Yu, and M. Nishimura (NARO Institute of Livestock and Grassland Science, Ibaraki, Japan) for their technical assistance.

\section{REFERENCES}

Ao, X., X. Zhang, X. Zhang, L. Shi, K. Zhao, J. Yu, L. Dong, Y. Cao, and Y. Cai. 2012. Identification of lactic acid bacteria in traditional fermented yak milk and evaluation of their application in fermented milk products. J. Dairy Sci. 95:1073-1084.

Bauchop, T., and S. R. Elsden. 1960. The growth of micro-organisms in relation to their energy supply. J. Gen. Microbiol. 23:457-469. 
Bhardwaj, A., H. Gupta, S. Kapila, G. Kaur, V. Shilpa, and R. K. Malik. 2010. Safety assessment and evaluation of probiotic potential of bacteriocinogenic Enterococcus faecium KH 24 strain under in vitro and in vivo conditions. Int. J. Food Microbiol. 141:156-164.

Botes, M., C. A. van Reenen, and L. M. T. Dicks. 2008. Evaluation of Enterococcus mundtii ST4SA and Lactobacillus plantarum 423 as probiotics by using a gastro-intestinal model with infant milk formulations as substrate. Int. J. Food Microbiol. 128:362-370.

Callon, C., J. L. Berdague, E. Dufour, and M. C. Montel. 2005. The effect of raw milk microbial flora on the sensory characteristics of Salers-type cheeses. J. Dairy Sci. 88:3840-3850.

Chammas, G. I., R. Saliba, G. Corrieu, and C. Beal. 2006. Characterization of lactic acid bacteria from fermented milk "Laban". Int. J. Food Microbiol. 110:52-61.

Cogan, T. M. 1981. Constitutive nature of the enzymes of citrate metabolism in Streptococcus lactis ssp. diacetylactis. J. Dairy Res. 48:489-495.

Dubois, M., K. A. Gilles, J. K. Hamilton, P. A. Rebers, and F. Smith. 1956. Colorimetric method for determination of sugars and related substrates. Anal. Chem. 28:350-356.

Faye, T., A. Tamburello, G. E. Vegarud, and S. Skeie. 2012. Survival of lactic acid bacteria from fermented milks in an in vitro digestion model exploiting sequential incubation in human gastric and duodenum juice. J. Dairy Sci. 95:558-566.

Frengova, G. I., E. D. Simova, D. M. Beshkova, and Z. I. Simov. 2000 Production and monomer composition of exopolysaccharides by yogurt starter cultures. Can. J. Microbiol. 46:1123-1127.

Garrigues, C., P. Louiere, N. D. Lindley, and M. Cocaign-Bousquet. 1997. Control of the shift from homolactic acid to mixed-acid fermentation in Lactococcus lactis: Predominant role of the NADH/ $\mathrm{NAD}^{+}$ratio. J. Bacteriol. 179:5282-5287.

Gibson, G. R., and M. B. Roberfroid. 1995. Dietary modulation of the human colonic microbiota: Introducing the concept of prebiotics. J. Nutr. 125:1401-1412.

Haarman, M., and J. Knol. 2006. Quantitative real-time PCR analysis of fecal Lactobacillus species in infants receiving a prebiotic infant formula. Appl. Environ. Microbiol. 72:2359-2365.

Iwata, E., H. Hotta, and M. Goto. 2009. The screening method of a bifidogenic dietary fiber extracted from inedible parts of vegetables. J. Nutr. Sci. Vitaminol. (Tokyo) 55:385-388.

Kimoto, H., K. Mizumachi, T. Okamoto, and J. Kurisaki. 2004a. New Lactococcus strain with immunomodulatory activity: Enhancement of Th1-type immune response. Microbiol. Immunol. 48:75-82.

Kimoto, H., M. Nomura, M. Kobayashi, T. Okamoto, and S. Ohmomo. 2004b. Identification and probiotic characteristics of Lactococcus strains from plant materials. Jpn. Agric. Res. Q. 38:111-117.

Kimoto, H., M. Nomura, and I. Suzuki. 1999. Growth energetics of Lactococcus lactis ssp. lactis biovar diacetylactis in cometabolism of citrate and glucose. Int. Dairy J. 9:857-863.
Kimoto-Nira, H., C. Suzuki, M. Kobayashi, K. Sasaki, J. Kurisaki, and K. Mizumachi. 2007. Anti-aging effect of a lactococcal strain: Analysis using senescence-accelerated mice. Br. J. Nutr. 98:11781186.

Klijn, N., A. H. Weerkamp, and W. M. de Vos. 1995. Genetic marking of Lactococcus lactis shows its survival in the human gastrointestinal tract. Appl. Environ. Microbiol. 61:2771-2774.

Kothari, S. L., V. K. N. Nambudrupad, and H. Laxminarayana. 1971. Studies on the associative growth of streptococci. Milchwissenschaft 26:415-418.

Lee, W.-K., H.-J. Lim, S.-Y. Kim, H. Kimoto, S. Ohmomo, Y. Tashiro, and H. Takebe. 2005. Hypocholesterolemic effect of Lactococcus lactis ssp. lactis biovar diacetylactis N7 and Lactococcus lactis ssp. lactis 527 strains in SD rats. Biosci. Microflora 24:11-16.

Loubiere, P., P. Salou, M. J. Leroy, N. D. Lindley, and A. Pareilleux. 1992. Electrogenic malate uptake and improved growth energetics of the malolactic bacterium Leuconostoc oenos grown on glucosemalate mixtures. J. Bacteriol. 174:5302-5308.

Marteau, P., and J.-C. Rambaud. 1993. Potential of using lactic acid bacteria for therapy and immunomodulation in man. FEMS Microbiol. Rev. 12:207-220.

Meers, J. L. 1973. Growth of bacteria in mixed cultures. Crit. Rev. Microbiol. 2:139-184.

Miyazaki, Y., S. Kamiya, T. Hanawa, M. Fukuda, H. Kawakami, H. Takahashi, and H. Yokota. 2010. Effect of probiotic bacterial strains of Lactobacillus, Bifidobacterium, and Enterococcus on enteroaggregative Escherichia coli. J. Infect. Chemother. 16:10-18.

Picon, A., and M. Nunez. 2007. Growth stimulation of a proteinase positive Lactococcus lactis strain by a proteinase negative Lactococcus lactis strain. Int. J. Food Microbiol. 119:308-313.

Russell, J. B., and G. M. Cook. 1995. Energetics of bacterial growth Balance of anabolic and catabolic reactions. Microbiol. Rev. 59:48-62.

Schmitt, P., and C. Divies. 1991. Co-metabolism of citrate and lactose by Leuconostoc mesenteroides ssp. cremoris. J. Ferment. Bioeng. 71:72-74.

Schmitt, P., C. Divies, and C. Merlot. 1990. Utilization of citrate by Leuconostoc mesenteroides ssp. cremoris in continuous culture. Biotechnol. Lett. 12:127-130.

Smart, J. B., and T. D. Thomas. 1987. Effect of oxygen on lactose metabolism in lactic streptococci. Appl. Environ. Microbiol. 53:533-541.

Starrenburg, M. J. C., and J. Hugenholtz. 1991. Citrate fermentation by Lactococcus and Leuconostoc spp. Appl. Environ. Microbiol. 57:3535-3540.

Westerfeld, W. W. 1945. A colorimetric determination of blood acetoin. J. Biol. Chem. 16:495-502. 